\title{
Solvent Control of Electron Transfer Dynamics
}

\author{
Erik R. Barthel, Ignacio B. Martini, Ernö Keszei, and Benjamin J. Schwartz
}

UCLA Department of Chemistry and Biochemistry, Los Angeles, CA 90095-1569 USA

Tel. (310) 206 4113; Fax (310) 2064038

E-mail: schwartz@chem.ucla.edu

Abstract. We investigate the charge-transfer-to-solvent (CTTS) dynamics of sodide in several solvents. Since this CTTS system has only electronic degrees of freedom, the dynamics provide a window into how solvent motions control electron transfer.

It is of great interest to understand how motions of solvent molecules control the dynamics of electron transfer (ET). Here we tackle this problem using the charge-transfer-to-solvent (CTTS) transition of sodide ( $\left.\mathrm{Na}^{-}\right)$[1]. Absorption of a visible photon by $\mathrm{Na}^{-}$produces a localized excited state bound only by the polarization of the solvent, from which solvent motions promote electron detachment. Since the CTTS band of $\mathrm{Na}^{-}$is similar in a variety of ethers and amines [2], we can use ultrafast spectroscopy to determine how the local solvent structure controls the dynamics of CTTS detachment. We find that there is a subps delayed detachment of the electron from the CTTS excited state $\left(\mathrm{Na}^{-*}\right)$ followed by back ET (geminate recombination) on a time scale of a few ps. For ether solvents, the back ET times correlate with solvent polarity, with more polar ethers having shorter back ET times. We also find that this trend does not hold in non-ether solvents, and there appears to be no correlation of the forward ET (detachment) time with solvent polarity. This points to the importance of local solvent structure in determining the CTTS detachment dynamics.

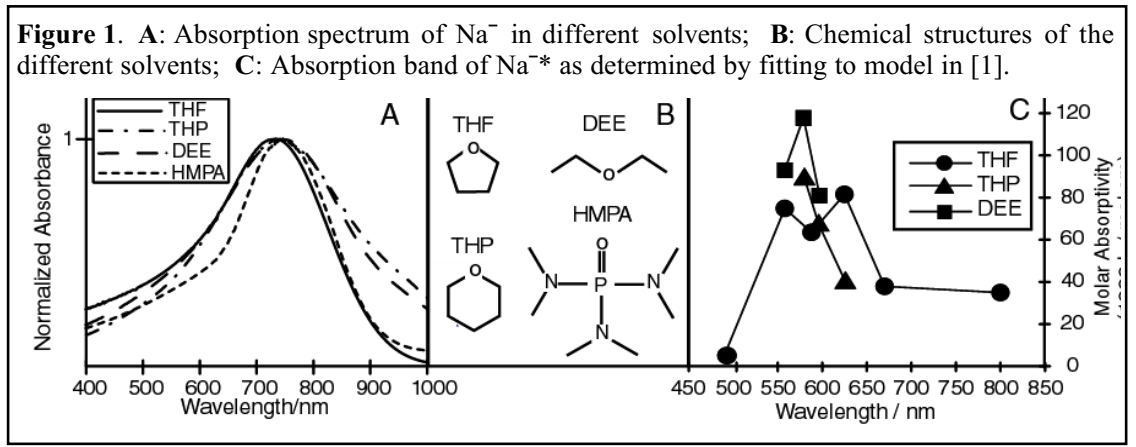

Figure 1A shows the absorption spectrum of the $\mathrm{Na}^{-}$CTTS transition in tetrahydrofuran (THF), tetrahydropyran (THP), diethyl ether (DEE), and hexamethylphosphoramide (HMPA); Figure 1B shows the chemical structures of each of these solvents. The spectrum of $\mathrm{Na}^{-}$is similar not only in the three ethers but also the more polar HMPA. The $\sim 2 \mu \mathrm{m}$ absorption of the solvated electron in HMPA is also similar to that in ethers. This is a result of the diffuse nature of the positive dipole of HMPA and the difficulty for large HMPA molecules to form cavities around negative particles with radii smaller than 4-5 $\AA$ [3], leading 
to a similar cavity size as for electrons in ethers. These factors also explain why the spectrum of $\mathrm{Na}^{-}$in HMPA is so similar to that in the ethers.

Figure $1 \mathrm{C}$ shows the visible transient absorption spectrum of the sodide CTTS excited state, $\mathrm{Na}^{-*}$, as determined by fitting pump-probe data to a detailed kinetic model presented previously [1]. This species has its absorption maximum near $590 \mathrm{~nm}$, the location of the sodium D line. The similarity of the spectrum in all solvents investigated supports our assignment of this absorption to a $\mathrm{Na}$ atom core that does not interact strongly with the surrounding solvent.[1]

Figure 2 shows pump-probe data for $\mathrm{Na}^{-}$excited at $\sim 500 \mathrm{~nm}$ and probed at both $\sim 580 \mathrm{~nm}$ and at $\sim 2 \mu \mathrm{m}$. The solid curves are fits to the data using our kinetic model [1]. Probing at $580 \mathrm{~nm}$ monitors both $\mathrm{Na}^{-*}$ and the ground state bleach of $\mathrm{Na}^{-}$, while probing at $2 \mu \mathrm{m}$ monitors solvated electrons. In the three ether solvents, the 580-nm transient absorption is instrument-limited, and then decays as the electron detaches from $\mathrm{Na}^{-*}$ and the solvent rushes in to destroy $\mathrm{Na}^{-*}$ and create a solvated $\mathrm{Na}$ atom. Although the ejection mechanism is qualitatively similar, it is clear that the time for the forward ET, as measured by the decay of the $\mathrm{Na}^{-*}$ absorption, is quite different in the three ethers.

This conclusion is also supported by the matching rise times for the solvated electron's absorption at $\sim 2 \mu \mathrm{m}$, which also reflect the $\mathrm{e}^{-}$detachment time. The back ET (geminate recombination) time scale is also different for each solvent. As shown in Table 1, the back ET times increase with decreasing solvent polarity for the ethers [4]. However, this trend does not include the highly polar solvent HMPA, in which the back ET time is considerably slower.

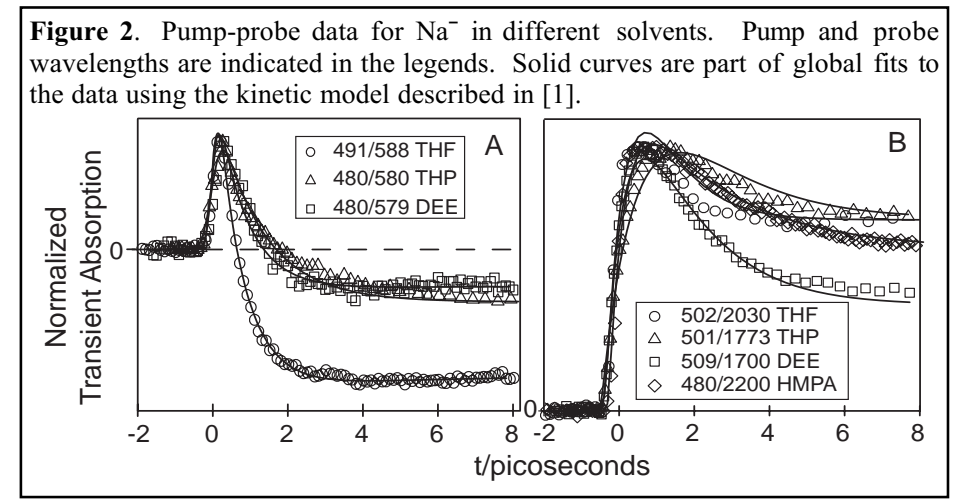

The trend in back ET times with solvent polarity, and the lack of such a trend for the forward ET times, suggests different mechanisms for the two ET processes. The initial photoexcitation of $\mathrm{Na}^{-}$produces a $p$-like state that must detach and localize into an $s$-like solvated electron. We believe that translational motions of the solvent molecules into the $p$-like angular node promote this detachment. In the fastest solvent, THF, inward translations of the nearly planar ring can be assisted by translation-rotation coupling, as seen previously in MD simulations [5]. In the next-fastest solvent, DEE, we believe that free rotation about the $\mathrm{C}-\mathrm{O}$ bond decreases the ability (relative to THF) of the first-shell molecules of this solvent to undergo inward translation. Finally, for slow THP, it is likely that packing of the solvent into chair-like structures makes it difficult for the first-shell solvent molecules to translate into the angular node, resulting in 
a slower response. This type of packing effect is also seen in the pronounced liquid structure for nearest neighbors in the related solvent, cyclohexane [6].

\begin{tabular}{|cccccc|}
\hline $\begin{array}{c}\text { Table 1. ET times for } \mathrm{Na}^{-} \text {in solvents with different dielectric properties; the } \\
\text { dielectric values are taken from [4]. The ET times have an uncertainty of } \pm 0.05 \text { ps. } \\
\text { solvent }\end{array} \quad$ abbreviation & $\boldsymbol{\varepsilon} / \varepsilon_{0}$ & $\boldsymbol{\mu} / \mathrm{D}$ & $\begin{array}{c}\text { ET Times (ps) } \\
\text { forward }\end{array}$ & reverse \\
diethyl ether & DEE & 4.42 & 1.15 & 0.82 & 1.53 \\
tetrahydropyran & THP & 5.68 & 1.63 & 1.26 & 1.38 \\
tetrahydrofuran & THF & 7.47 & 1.75 & 0.70 & 0.78 \\
hexamethylphosphoramide & HMPA & 29.00 & 5.55 & 0.41 & 2.51 \\
\hline
\end{tabular}

In contrast to detachment, the fast back ET is due to the recombination of contact pairs [1] in which the sodium atom is in the electron's first solvation shell. The recombination progresses via a nonadiabatic transition, the rate of which depends on the overlap of the electron's wave function with its geminate $\mathrm{Na}$ atom partner. This is why the macroscopic dielectric properties of the solvent become important in stabilizing the contact pair: the less polar the solvent, the better solvated the neutral sodium atom and the less favorable the back ET.

The large differences in the CTTS dynamics in very polar HMPA likely are due to a different molecular solvent structure. The bulky nature of this solvent creates large cavities even before electron detachment [3]. Thus, the forward detachment can proceed rapidly since little solvent translational motion is needed, and the unusually high stability of the newly-formed solvated electron cavity could then result in the observed slow back ET time

Overall, the results show that it is not sufficient to consider only the dielectric properties of a solvent when considering ET dynamics. The local molecular structure of a solvent can have direct effects on the motions that control the dynamics of ET. For the $\mathrm{Na}^{-}$CTTS reaction in weakly polar ether solvents, the forward ET time is dictated primarily by the local solvent structure, whereas the back ET depends mostly on solvent polarity. For the bulky polar solvent HMPA, the slow back ET reaction likely is due to the tendency for the solvent to form unusually stable cavities for the solvated electron.

\section{References}

[1] E. R. Barthel, I. B. Martini, B. J. Schwartz, "How Does the Solvent Control ET? Experimental and Theoretical Studies of the Simplest Charge Transfer Reaction," J. Phys. Chem. B 105, 12330-41 (2001).

[2] M. T. Lok, F. J. Tehan, J. L. Dye, "Spectra of $\mathrm{Na}^{-}, \mathrm{K}^{-}$, and e-solv in Amines and Ethers," J. Phys. Chem. 76, 2975-81 (1972).

[3] E. A. Shaede, L. M. Dorfman, G. J. Flynn, D. C. Walker, "Spectrum, Kinetics, and Radiation Chemical Yield of Solvated Electrons in Hexamethylphosphoric Triamide," Can. J. Chem. 51, 3905-3913 (1973).

[4] C. Laurence, P. Nicolet, M. T. Dalati, J. -L. M. Abboud, R. Notario, "The Empirical Treatment of Solvent-Solute Interactions: 15 Years of $\pi^{*}$," J. Phys. Chem. 98, 5807-5816 (1994).

[5] V. Tran, B. J. Schwartz, "Role of Nonpolar Forces in Aqueous Solvation: Computer Simulation Study of Solvation Dynamics in Water Following Changes in Solute Size, Shape, and Charge," $J$. Phys. Chem. B 103, 5570-80 (1999).

[6] J. G. Harris, F. H. Stillinger, "Isomerization and Inherent Structure in Liquids. A Molecular Dynamics Study of Liquid Cyclohexane," J. Chem. Phys. 95, 5953-65 (1991). 